\title{
AÇÕES RÁPIDAS DA TRIIODOTIRONINA (T3) SOBRE A EXPRESSÃO E SECREÇÃO DE TSH: NOVOS MECANISMOS ENVOLVIDOS NO FEEDBACK NEGATIVO
}

Tese apresentada ao Programa de Pós-Graduação em Fisiologia Humana do Instituto de Ciências Biomédicas da Universidade de São Paulo, para obtenção do Título de Doutor em Ciências.

Área de concentração: Fisiologia Humana

Orientadora: Profa. Dra. Maria Tereza Nunes

Versão original 


\section{RESUMO}

Bargi-Souza P. Ações rápidas da Triiodotironina (T3) sobre a expressão e secreção de TSH: novos mecanismos envolvidos no feedback negativo. [Tese (Doutorado em Ciências)]. São Paulo: Instituto de Ciências Biomédicas, Universidade de São Paulo; 2015.

O hormônio tireotrófico (TSH) é o principal regulador da síntese e da secreção dos hormônios tiroidianos (HTs), os quais exercem um mecanismo de feedback negativo na hipófise reduzindo a síntese das cadeias beta (Tshb) e alfa (CGA - Glycoprotein hormones Alpha Chain) por meio de mecanismos que envolvem modificações na transcrição dos genes que codificam essas proteínas (ações genômicas). Recentemente tem aumentado o número de evidências de que, em paralelo as ações genômicas clássicas, algumas ações dos HTs são desencadeadas na presença de inibidores da transcrição gênica e em curto espaço de tempo, caracterizando-se assim as ações não genômicas dos HTs. Este estudo tem como objetivos: 1) avaliar as possíveis ações não genômicas do T3 sobre a expressão, processamento póstranscricional e tradução das subunidades Cga e Tshb que compõem o TSH em cultura de células TaT1; 2) caracterizar as vias envolvidas no bloqueio da secreção de TSH desencadeado rapidamente pelo T3 em cultura primária de células da adenohipófise e; 3) avaliar a participação do T3 na mobilização intracelular dos cátions cálcio e magnésio em cortes de hipófise. Por meio das metodologias de RT-qPCR, RACE-PAT e perfil polissomal obervamos que o T3reduziu rapidamente o conteúdo de mRNA de Tshb mesmo na presença do bloqueador da transcrição gênica $\mathrm{DRB}$, o comprimento da sua cauda poli(A), via interação com a integrina $\alpha \mathrm{V} \beta 3$, bem como sua interação com ribossomos, reduzindo assim a taxa de tradução deste transcrito e consequentemente a síntese de TSHB. Com o uso de inibidores específicos para PI3K, bem como do hexapeptídeo RGD, que impede a interação do T3 com a integrina de membrana $\alpha \mathrm{V} \beta 3$, observamos por meio de Western blotting e dosagem hormonal por ELISA, que o bloqueio rápido da secreção de TSH induzido pelo T3 foi abolido, indicando que o aumento desta glicoproteína no meio intracelular e a redução do seu conteúdo no meio extracelular induzido pelo T3 ocorre por vias dependentes da PI3K e da integrina $\alpha \mathrm{V} \beta 3$. A redução de cálcio livre no sistema, pelo uso de EGTA no meio de cultura, não alterou o efeito do T3. Ainda, observamos em cortes de hipófise que o T3 não altera a mobilização intracelular de cálcio em tireotrofos, porém, aumenta a concentração intracelular de magnésio em 16\% das células da adenohipófise, sugerindo a participação deste cátion na inibição da secreção de TSH induzida pelo T3, atuando diretamente nos tireotrofos ou por mecanismos parácrinos. Estes dados demonstram a existência de um mecanismo adicional de redução da síntese/secreção de $\mathrm{TSH}$, desencadeado em poucos minutos pelo T3, por meio de sua interação com a integrina $\alpha \mathrm{V} \beta 3$ na membrana plasmática, caracterizando uma ação não genômica deste hormônio sobre o controle da sua própria síntese e secreção. Este mecanismo se soma ao já reconhecido efeito de feedback negativo via controle da taxa de transcrição gênica que ele desencadeia.

Palavras-chave: Hormônio tireoidiano. Tirotrofina (TSH). Secreção. Ações não genômicas. Poliadenilação. Tireotrofo. 


\begin{abstract}
Bargi-Souza P. Rapid actions of Triiodothyronine (T3) on TSH expression and secretion: new mechanisms involved in the negative feedback. [Ph. D. thesis (Science)]. São Paulo: biomedical Science Institute, University of São Paulo; 2015.

Thyrotropin (TSH) is the main regulator of the thyroid hormones (HTs) synthesis and secretion, which in turn exert a negative feedback mechanism on the $T s h b$ and Cga (Glycoprotein hormones alpha chain) mRNA expressions in the pituitary gland by reducing their transcription rate (genomic actions). In parallel to the classical genomic actions of HTs, some of its effects are triggered in the presence of gene transcription inhibitors in short period of time (seconds to minutes), characterizing the non genomic actions of HTs. Rapid effects of T3 have been characterized in Tshb expression and TSH secretion, for this reason, the present study aimed to: 1) evaluate the possible non-genomic actions of T3 on the Cga and Tshb expression, post-transcriptional processing and translation in T $\alpha \mathrm{T} 1$ cells; 2) characterize the pathways involved in the rapid inhibition of the TSH secretion induced by T3 in primary cultures of anterior pituitary cells and; 3) evaluate the participation of T3 on calcium and magnesium intracellular mobilization in slices of pituitary of hypothyroid rats. Using Real Time PCR, RACE-PAT and polysomal profile methods, we have observed that T3 quickly reduced the $T s h b$ mRNA content and its poly(A) tail length, even in the presence of DRB, an inhibitor of gene transcription, via interaction with the integrin $\alpha \mathrm{V} \beta 3$, as well as, its interaction with the ribosomes, reducing the transcript translation rate, and consequently the TSHB synthesis. Using specific PI3K inhibitor, as well the hexapeptide RGD, which prevents the interaction between $\mathrm{T} 3$ and $\alpha \mathrm{V} \beta 3$ integrin, we have observed by Western blotting and ELISA assay, that blockade of TSH secretion induced by T3 was abolished, indicating that the increase in the intracellular content of TSHB and its reduction in the extracellular medium by T3 occurs by $\alpha \mathrm{V} \beta 3$ integrin- and PI3K-dependent mechanisms. The effects of T3 on TSH secretion were not changed in the presence of EGTA in the culture media, which reduces the free calcium in our experimental condition. Moreover, the experiments of calcium and magnesium mobilization in pituitary slices of hypothyroid rats have demonstrated that T3 does not change the mobilization and concentration of intracellular calcium in thyrotrophs, but, it increases the intracellular concentration of magnesium in $16 \%$, suggesting the involvement of this ion in the inhibition of TSH secretion by direct or paracrine mechanisms. Therefore, we propose the existence of an additional mechanism that decreases the TSH synthesis/secretion, triggered in few minutes by $\mathrm{T} 3$, through its interaction with $\alpha \mathrm{V} \beta 3$ integrin at the plasma membrane, featuring a non genomic action of this thyroid hormone on its own synthesis and secretion. This mechanism, which is quickly triggered by T3, can be added to the already well known negative feedback regulation induced by T3 via control of gene transcription.
\end{abstract}

Keywords: Thyroid hormone. Thyrotropin (TSH). Secretion. Non genomic actions. Polyadenylation. Thyrotroph. 


\section{INTRODUÇÃO}

A adenohipófise, ou hipófise anterior, possui um papel central na regulação da função endócrina através da produção e secreção de hormônios tróficos, processos que estão sob regulação hipotalâmica. Os hormônios tróficos produzidos na adenohipófise são liberados na circulação sanguínea e a partir desta atingem os órgãos alvos gerando uma resposta fisiológica que frequentemente está associada com a liberação de hormônios produzidos por glândulas/órgãos alvo (1).

Os tipos celulares presentes na adenohipófise compreendem os somatotrofos, lactotrofos, corticotrofos, gonadotrofos e tireotrofos que sintetizam e secretam o hormônio do crescimento $(\mathrm{GH})$, a prolactina ( $\mathrm{Prl})$, o hormônio adrenocorticotrófico (ACTH), hormônio luteinizante $(\mathrm{LH})$ e folículo estimulante (FSH) e o hormônio tireotrófico (TSH), respectivamente. Deste modo, a adenohipófise participa da regulação de importantes funções vitais como crescimento, reprodução e metabolismo (2).

O hormônio tireotrófico (TSH), também denominado tireotrofina, é uma glicoproteína de $28 \mathrm{kDa}$ e, assim como as demais glicoproteínas hipofisárias (LH e FSH), é composta por duas diferentes subunidades, alfa (CGA - Glycoprotein hormones Alpha Chain) e beta (TSHB), associadas por ligação não covalente (3). Sua síntese e secreção ocorrem nos tireotrofos, tipo celular que compreende cerca de $5-10 \%$ da hipófise anterior em ratos.

Ocupando uma posição central no eixo hipotálamo-hipófise-tiróide, o TSH é responsável pela manutenção apropriada da concentração dos hormônios tiroidianos (HTs), a triiodotironina (T3) e a tiroxina (T4), na circulação (4), atuando em várias etapas da síntese e secreção destes, bem como no metabolismo da glândula tiróide.

Os HTs, por sua vez, exercem seus efeitos biológicos, através do controle da expressão de genes específicos, o que leva ao aumento ou diminuição da síntese de proteínas envolvidas com o metabolismo, crescimento e desenvolvimento. Essas ações, denominadas de genômicas, nucleares ou transcricionais, decorrem da interação do T3 com seus receptores, os quais se apresentam associados às sequências específicas localizadas na região promotora de seus genes-alvo, conhecidas como elementos responsivos aos HTs (TREs) (5).

A maioria desses genes tem sua transcrição aumentada pelo T3, como é o caso do gene que codifica o GH (6), outros, contudo, têm sua expressão reprimida pelo T3, como é o caso dos genes que codificam as subunidades alfa (Cga) (7) e beta do TSH (Tshb) (8) e o Trh (Hormônio Liberador da Tireotrofina) (5). Este mecanismo de ação requer um tempo relativamente longo, de horas ou até dias, para que a resposta hormonal seja desencadeada. 
Por outro lado, algumas ações dos HTs são desencadeadas em curto espaço de tempo (segundos a minutos) e na presença de inibidores da transcrição gênica, o que indica que eles exercem também ações extranucleares, também denominadas de não genômicas ou póstranscricionais. Estas ações resultam em alterações no fluxo de íons e de glicose, em componentes do citoesqueleto, na atividade de quinases específicas, na estabilidade de alguns transcritos, ou ainda em aumento da atividade e inserção de proteínas na membrana plasmática, como a Na,K-ATPase (9-14).

Parte das ações não genômicas ocorre após a passagem dos HTs pela membrana plasmática e sua interação com quinases citossólicas, nas mitocôndrias ou no retículo endoplasmático, enquanto outra parcela das ações extranucleares dos HTs ocorre após a interação destes hormônios com um sítio ligante, recentemente descrito na membrana celular, localizado próximo ao domínio extracelular da proteína integrina $\alpha v \beta 3$, que reconhece proteínas de matriz que apresentam o domínio RGD $(10,15)$.

\subsection{Ações dos hormônios tiroidianos sobre a síntese e secreção de TSH - mecanismo de feedback negativo}

Estudos anteriores demonstraram que os HTs atuam na hipófise e no hipotálamo reduzindo a síntese e secreção de TSH (tanto de CGA quanto de TSHB), e TRH, respectivamente, por meio de ações diretas no processo de transcrição gênica (16-18). Estas ações são desencadeadas pelo T3, principalmente o gerado na hipófise e hipotálamo, a partir da desiodação intracelular do T4 circulante, e decorrem da interação do T3 com os receptores de HTs (THRs) presentes na região promotora dos genes que codificam as cadeias alfa e beta do TSH e o TRH (19).

Recentes estudos desenvolvidos in vitro por Chiamolera e colaboradores (20) evidenciaram que próximo à região promotora do gene do Tshb há uma elevada concentração de THRB (isoforma beta do THR), e, utilizando-se de ferramentas como shRNA (small hairpin RNA), demonstraram que esta isoforma é essencial para as ações exercidas pelo T3 que caracterizam o feedback negativo sobre a expressão do Tshb, mesmo frente ao estímulo com TRH. Esses dados corroboram estudos anteriores, que já apontavam essa isoforma como a principal envolvida no mecanismo de feedback negativo exercido pelo T3 sobre o gene do Tshb (5).

Deste modo, quando há uma elevação da concentração plasmática de HT, ocorre maior inibição da expressão de Cga, Tshb e Trh, e quando há redução dos HTs no plasma, observa- 
se o oposto, condições em que a tiróide tem sua atividade reduzida ou aumentada, respectivamente, o que caracteriza o mecanismo de feedback negativo desencadeado pelos HTs circulantes (21-23).

Somado a ação genômica dos HTs sobre o TSH, dados presentes na literatura demonstraram que após 8 h do tratamento com T3, ocorre diminuição da estabilidade e da meia vida (de 24 para 9 h) do mRNA do Tshb, evento associado a uma redução no grau de poliadenilação deste transcrito, caracterizando uma regulação da expressão desta subunidade a nível pós-transcricional, desencadeada pelo T3 (24).

Em continuidade ao trabalho sobre possíveis alterações pós-transcricionais do T3 sobre o mRNA de Tshb, LEEDMAN e colaboradores (25) isolaram uma proteína que se liga na porção 3'UTR (região não tradutível) deste mRNA desestabilizando-o. Ainda neste estudo, foi observado que esta proteína é regulada pelo T3, e que poderia estar envolvida na regulação não genômica da estabilidade do mRNA de Tshb pelo HT.

Ainda, sabe-se que tanto o ácido triiodotiroacético (Triac) bem como o T3 e o T4 reduzem rapidamente (15 min) a secreção de TSH em células de adenohipófise estimuladas com TRH, observando-se, em apenas duas horas, uma redução de 17\% frente à administração de T3 e de $9 \%$ para T4 (26).

Esses dados, somados às alterações no conteúdo e estabilidade do mRNA de Tshb, sugerem que mecanismos pós-transcricionais ou não genômicos estejam sendo desencadeados pelos HTs, modificando a síntese como também a secreção de TSH.

\subsection{Ações não genômicas dos HTs: Contribuição do nosso laboratório}

Enquanto vários estudos vêm sendo desenvolvidos para melhor compreensão do mecanismo de ação genômica dos HTs sobre a expressão gênica de Tshb poucos trabalhos existem sobre as suas possíveis ações não genômicas e, considerando a importância de que respostas rápidas sejam desencadeadas frente às flutuações da concentração dos HTs na circulação, torna-se importante que tal avaliação seja feita.

Nosso laboratório vem despendendo esforços para a identificação de possíveis ações não genômicas dos HTs. Trabalhos focados neste tema demonstraram que ratos hipotiroideos tratados agudamente (30 min) com doses supra-fisiológicas de T3 (100 $\mu \mathrm{g} / 100$ g PC) apresentaram uma redução de $40 \%$ no grau de poliadenilação do mRNA de Tshb, sem que seu conteúdo total fosse alterado, e uma diminuição no conteúdo deste associado aos 
ribossomos, caracterizando uma ação pós-transcricional deste hormônio reduzindo a síntese de TSHB (13).

Quando os mesmos procedimentos foram realizados utilizando-se dose fisiológica de T3 (0,3 $\mu \mathrm{g} / 100$ g PC) (27) não foram observadas alterações no comprimento da cauda poli(A) dos transcritos, o que indica que, dependendo da dose de T3 administrada, há uma resposta celular diversa. Estas respostas, aparentemente discrepantes, possivelmente resultam da ativação simultânea de diferentes vias/mecanismos pelo T3 num curto período de tempo (30 min), já que os diferentes sítios ligantes de HT, supostamente, apresentam graus distintos de afinidade a eles, gerando diferentes respostas frente à diferentes doses.

Mais recentemente, um trabalho publicado pelo nosso grupo também demonstrou que no hipotiroidismo, o conteúdo de TSHB é escasso no citoplasma de tireotrofos e suas vesículas de secreção apresentam-se distribuídas, principalmente, próximo à membrana plasmática e no interior de vasos sanguíneos, o que está de acordo com os altos níveis de secreção do TSH observados nesta condição experimental. Após o tratamento com T3 por 30 min (em doses fisiológicas ou suprafisiológicas) houve um aumento no contéudo de TSHB e uma redistribuição dos seus grânulos de secreção, de modo que a marcação deste hormônio apresentou-se dispersa pelo citoplasma e não mais próxima à membrana plasmática ou no interior de vasos sanguíneos, dado indicativo de um rápido bloqueio na secreção de TSH (28) (Apêndice).

Assim, podemos afirmar que tanto a síntese como a secreção são rapidamente reguladas pelo T3. Contudo, os mecanismos e as vias pelos quais o T3 promove essas alterações, em um curtíssimo espaço de tempo ainda são desconhecidos.

Para uma abordagem mais aprofundada desses efeitos, o presente estudo utilizou cultura de células tireotróficas T $\alpha \mathrm{T} 1$, modelo que permite um maior controle sobre os parâmetros externos, bem como cultura primária de células da adenohipófise de animais hipotiroideos. Estes modelos possibilitaram o uso de bloqueadores de transcrição gênica e inibidores de proteínas envolvidas em vias de sinalização específicas para a identificação do mecanismo pelo qual o T3 poderia promover seus rápidos efeitos sobre a síntese e secreção do TSH.

\subsection{Linhagem celular de tireotrofos - Células T $\alpha \mathrm{T} 1$ de camundongos}

Recentemente, ALARID e colaboradores (29) imortalizaram células da hipófise de camundongos transgênicos, em diferentes estágios de diferenciação, obtendo uma linhagem de tireotrofo que expressa tanto a subunidade alfa quanto a beta do TSH e o fator de 
transcrição Pit-1, que está relacionado com o desenvolvimento e sobrevivência dos tireotrofos presentes na pars distalis da adenohipófise.

Estudos com estas células tireotróficas imortalizadas, linhagem T $\alpha \mathrm{T} 1$, demonstraram que as mesmas apresentam os THRs nas isoformas THRB1 e THRB2, além do receptor RXR $\gamma$ que sofre heterodimerização com os THRs, de modo a possibilitar que as ações genômicas dos HTs sejam desencadeadas e estudadas (4). Este estudo também mostrou que as células T $\alpha \mathrm{T} 1$ respondem ao feedback negativo exercido pelo T3 sobre a expressão do mRNA de Tshb, porém, não foi observada qualquer ação sobre o transcrito Cga e sobre a meia-vida do mRNA do Tshb.

Apesar destas particularidades, as células T $\alpha \mathrm{T} 1$ apresentam características de células tireotróficas muito preservadas, como a expressão do fator de transcrição Pit-1 nas isoformas Pit-1 $\beta$ e Pit-T e é, portanto, o modelo mais próximo do real e viável para estudos biológicos. Por essa razão utilizamos esta linhagem celular, com o intuito de melhor caracterizar ações rápidas e possivelmente pós transcricionais sobre a síntese e processamento do mRNA de Tshb na linhagem celular T $\alpha \mathrm{T} 1$.

Problemas na detecção da proteína TSHB nesta linhagem celular nos levaram a utilizar o modelo de cultura primária de células da adenohipófise de animais hipotiroideos para os estudos envolvendo a caracterização das possíveis vias acionadas pelo T3 que resultariam na inibição da secreção do TSH.

Assim, o presente estudo teve como objetivo caracterizar as ações rápidas do T3 sobre a síntese, processamento, tradução e secreção de TSH em modelos de cultura primária de células da adenohipófise e em cultura de tireotrofos T $\alpha \mathrm{T} 1$, e deste modo, aprofundar o conhecimento do mecanismo de feedback negativo exercido por esse hormônio num componente central do eixo hipotálamo-hipófise-tireóide (HPT), que é o tireotrofo. 


\section{CONCLUSÕES}

Diante dos resultados obtidos no presente estudo, podemos concluir que, somado às ações genômicas inibitórias sobre a transcrição gênica da subunidade beta do TSH, o T3 por intermédio da sua interação com a integrina $\alpha \mathrm{V} \beta 3$ :

1) Reduz o conteúdo e o comprimento da cauda poli(A) do mRNA de Tshb;

2) Reduz o conteúdo de Tshb presente nos polissomos e consequentemente a taxa de tradução de TSHB;

3) Aumenta o conteúdo intracelular de TSHB;

4) Reduz a secreção de TSH, efeito este dependente da atividade de PI3K;

5) Aumenta a concentração intracelular de magnésio em 16\% das células hipofisárias possivelmente via PI3K e PLC $\gamma$;

6) Não altera a concentração intracelular de cálcio em tireotrofos.

Estes dados evidenciam pela primeira vez a existência de um mecanismo adicional pelo qual o T3 interage com a integrina $\alpha \mathrm{V} \beta 3$ e promove uma redução na síntese e secreção de TSH, em poucos minutos, caracterizando uma ação não genômica, que se soma ao já reconhecido efeito de feedback negativo que ele desencadeia. 


\section{REFERÊNCIAS}

1. Molina PE. Anterior pituitary gland. Endocrine physiology. United States of America: The McGraw-Hill Companies; 2006. p. 45.

2. Kutchai HC. Receptores de membrana, segundos mensageiros e vias de transdução de sinais. In: Berne RM, editor. Fisiologia. Rio de Janeiro: Elsiever Editora 2004. p. 65.

3. Pierce JG, Parsons TF. Glycoprotein Hormones: Structure and Function. Annual Review of Biochemistry. 1981;50:465-95.

4. Yusta B, Alarid ET, Gordon DF, Ridgway EC, Mellon PL. The Thyrotropin BetaSubunit Gene Is Repressed by Thyroid Hormone in a Novel Thyrotrope Cell Line, Mouse T Alphat1 Cells. Endocrinology. 1998 Nov;139(11):4476-82.

5. Shibusawa N, Hollenberg AN, Wondisford FE. Thyroid Hormone Receptor DNA Binding Is Required for Both Positive and Negative Gene Regulation. The Journal of Biological Chemistry. 2003 Jan 10;278(2):732-8.

6. Murphy D, Pardy K, Seah V, Carter D. Posttranscriptional Regulation of Rat Growth Hormone Gene Expression: Increased Message Stability and Nuclear Polyadenylation Accompany Thyroid Hormone Depletion. Molecular and Cellular Biology. 1992 Jun;12(6):2624-32.

7. Shupnik MA, Chin WW, Ross DS, Downing MF, Habener JF, Ridgway EC. Regulation by Thyroxine of the Mrna Encoding the Alpha Subunit of Mouse Thyrotropin. The Journal of Biological Chemistry. 1983 Dec 25;258(24):15120-4.

8. Staton JM, Leedman PJ. Posttranscriptional Regulation of Thyrotropin Beta-Subunit Messenger Ribonucleic Acid by Thyroid Hormone in Murine Thyrotrope Tumor Cells: A Conserved Mechanism across Species. Endocrinology. 1998 Mar;139(3):1093-100.

9. Davis PJ, Davis FB. Nongenomic Actions of Thyroid Hormone. Thyroid. 1996 Oct;6(5):497-504.

10. Davis PJ, Davis FB, Cody V. Membrane Receptors Mediating Thyroid Hormone Action. Trends in Endocrinology and Metabolism: TEM. 2005 Nov;16(9):429-35.

11. Silva FG, Giannocco G, Santos MF, Nunes MT. Thyroid Hormone Induction of Actin Polymerization in Somatotrophs of Hypothyroid Rats: Potential Repercussions in Growth Hormone Synthesis and Secretion. Endocrinology. 2006 Dec;147(12):5777-85.

12. Silva FG, Giannocco G, Luchessi AD, Curi R, Nunes MT. T3 Acutely Increases Gh Mrna Translation Rate and Gh Secretion in Hypothyroid Rats. Molecular and Cellular Endocrinology. 2010 Apr 12;317(1-2):1-7.

13. Goulart-Silva F, de Souza PB, Nunes MT. T3 Rapidly Modulates Tshbeta Mrna Stability and Translational Rate in the Pituitary of Hypothyroid Rats. Molecular and Cellular Endocrinology. 2011 Jan 30;332(1-2):277-82.

14. Davis PJ, Leonard JL, Davis FB. Mechanisms of Nongenomic Actions of Thyroid Hormone. Frontiers in Neuroendocrinology. 2008 May;29(2):211-8. 
15. Cody V, Davis PJ, Davis FB. Molecular Modeling of the Thyroid Hormone Interactions with Alpha V Beta 3 Integrin. Steroids. 2007 Feb;72(2):165-70.

16. Shupnik MA, Ridgway EC. Triiodothyronine Rapidly Decreases Transcription of the Thyrotropin Subunit Genes in Thyrotropic Tumor Explants. Endocrinology. 1985 Nov;117(5):1940-6.

17. Chin WW, Carr FE, Burnside J, Darling DS. Thyroid Hormone Regulation of Thyrotropin Gene Expression. Recent Progress in Hormone Research. 1993;48:393-414.

18. O'Shea PJ, Williams GR. Insight into the Physiological Actions of Thyroid Hormone Receptors from Genetically Modified Mice. The Journal of Endocrinology. 2002 Dec;175(3):553-70.

19. Chin WW, Shupnik MA, Ross DS, Habener JF, Ridgway EC. Regulation of the Alpha and Thyrotropin Beta-Subunit Messenger Ribonucleic Acids by Thyroid Hormones. Endocrinology. 1985 Mar;116(3):873-8.

20. Chiamolera MI, Sidhaye AR, Matsumoto S, He Q, Hashimoto K, Ortiga-Carvalho TM, et al. Fundamentally Distinct Roles of Thyroid Hormone Receptor Isoforms in a Thyrotroph Cell Line Are Due to Differential DNA Binding. Molecular Endocrinology. 2012 Jun;26(6):926-39.

21. Sugimoto K, Mori K, Uchida K, Kobayashi D, Itoi K. Quantitative Analysis of Thyroid-Stimulating Hormone Messenger Rna and Heterogeneous Nuclear Rna in Hypothyroid Rats. Brain Research Bulletin. 2007 Sep 14;74(1-3):142-6.

22. Ross DS, Ellis MF, Ridgway EC. Acute Thyroid Hormone Withdrawal Rapidly Increases the Thyrotropin Beta and Alpha-Subunit Messenger Ribonucleic Acids in Mouse Thyrotropic Tumors. Endocrinology. 1986 Mar;118(3):1006-10.

23. Carr FE, Need LR, Chin WW. Isolation and Characterization of the Rat Thyrotropin Beta-Subunit Gene. Differential Regulation of Two Transcriptional Start Sites by Thyroid Hormone. The Journal of Biological Chemistry. 1987 Jan 25;262(3):981-7.

24. Krane IM, Spindel ER, Chin WW. Thyroid Hormone Decreases the Stability and the Poly(a) Tract Length of Rat Thyrotropin Beta-Subunit Messenger Rna. Molecular Endocrinology. 1991 Apr;5(4):469-75.

25. Leedman PJ, Stein AR, Chin WW. Regulated Specific Protein Binding to a Conserved Region of the 3'-Untranslated Region of Thyrotropin Beta-Subunit Mrna. Molecular Endocrinology. 1995 Mar;9(3):375-87.

26. Everts ME, Visser TJ, Moerings EP, Docter R, van Toor H, Tempelaars AM, et al. Uptake of Triiodothyroacetic Acid and Its Effect on Thyrotropin Secretion in Cultured Anterior Pituitary Cells. Endocrinology. 1994 Dec;135(6):2700-7.

27. Dillmann WH, Berry S, Alexander NM. A Physiological Dose of Triiodothyronine Normalizes Cardiac Myosin Adenosine Triphosphatase Activity and Changes Myosin Isoenzyme Distribution in Semistarved Rats. Endocrinology. 1983 Jun;112(6):2081-7. 
28. Bargi-Souza P, Romano RM, Salgado Rde M, Goulart-Silva F, Brunetto EL, Tenorio Zorn TM, et al. Triiodothyronine Rapidly Alters the Tsh Content and the Secretory Granules Distribution in Male Rat Thyrotrophs by a Cytoskeleton Rearrangement-Independent Mechanism. Endocrinology. 2013 Dec;154(12):4908-18.

29. Alarid ET, Windle JJ, Whyte DB, Mellon PL. Immortalization of Pituitary Cells at Discrete Stages of Development by Directed Oncogenesis in Transgenic Mice. Development (Cambridge, England). 1996 Oct;122(10):3319-29.

30. Nicoletti I, Migliorati G, Pagliacci MC, Grignani F, Riccardi C. A Rapid and Simple Method for Measuring Thymocyte Apoptosis by Propidium Iodide Staining and Flow Cytometry. Journal of Immunological Methods. 1991 Jun 3;139(2):271-9.

31. Bargi-Souza P, Kucka M, Bjelobaba I, Tomic M, Janjic MM, Nunes MT, et al. Loss of Basal and Trh-Stimulated Tshb Expression in Dispersed Pituitary Cells. Endocrinology. 2015 Jan;1(1):242-54.

32. Kretschmannova K, Kucka M, Gonzalez-Iglesias AE, Stojilkovic SS. The Expression and Role of Hyperpolarization-Activated and Cyclic Nucleotide-Gated Channels in Endocrine Anterior Pituitary Cells. Molecular Endocrinology. 2012 Jan;26(1):153-64.

33. Chomczynski P, Sacchi N. Single-Step Method of Rna Isolation by Acid Guanidinium Thiocyanate-Phenol-Chloroform Extraction. Analytical Biochemistry. 1987 Apr;162(1):1569.

34. Chomczynski P, Sacchi N. The Single-Step Method of Rna Isolation by Acid Guanidinium Thiocyanate-Phenol-Chloroform Extraction: Twenty-Something Years On. Nature Protocols. 2006;1(2):581-5.

35. Pfaffl MW. A New Mathematical Model for Relative Quantification in Real-Time RtPcr. Nucleic Acids Research. 2001 May 1;29(9):e45.

36. Salles FJ, Richards WG, Strickland S. Assaying the Polyadenylation State of mRNAs. Methods (San Diego, Calif). 1999 Jan;17(1):38-45.

37. Bradford MM. A Rapid and Sensitive Method for the Quantitation of Microgram Quantities of Protein Utilizing the Principle of Protein-Dye Binding. Analytical Biochemistry. 1976 May 7;72:248-54.

38. Dussault Aa PM. Rapid and Simple Comparison of Messenger Rna Levels Using Real-Time Pcr. Biological Procedures online. 2006;8:1-10.

39. Dupre SM, Guissouma H, Flamant F, Seugnet I, Scanlan TS, Baxter JD, et al. Both Thyroid Hormone Receptor (Tr)Beta 1 and Tr Beta 2 Isoforms Contribute to the Regulation of Hypothalamic Thyrotropin-Releasing Hormone. Endocrinology. 2004 May;145(5):233745.

40. Zoeller RT, Tan SW, Tyl RW. General Background on the Hypothalamic-PituitaryThyroid (Hpt) Axis. Critical Reviews in Toxicology. 2007 Jan-Feb;37(1-2):11-53.

41. Volpato CB, Nunes MT. Role of Thyroid Hormone in the Control of Growth Hormone Gene Expression. Brazilian journal of medical and biological research $=$ Revista brasileira de 
pesquisas medicas e biologicas / Sociedade Brasileira de Biofisica [et al]. 1994 May;27(5):1269-72.

42. Gallie DR. A Tale of Two Termini: A Functional Interaction between the Termini of an Mrna Is a Prerequisite for Efficient Translation Initiation. Gene. 1998 Aug 17;216(1):1-11.

43. Yankulov K, Yamashita K, Roy R, Egly JM, Bentley DL. The Transcriptional Elongation Inhibitor 5,6-Dichloro-1-Beta-D-Ribofuranosylbenzimidazole Inhibits Transcription Factor Iih-Associated Protein Kinase. The Journal of biological chemistry. 1995 Oct 13;270(41):23922-5.

44. te Poele RH, Okorokov AL, Joel SP. Rna Synthesis Block by 5, 6-Dichloro-1-Beta-DRibofuranosylbenzimidazole (Drb) Triggers P53-Dependent Apoptosis in Human Colon Carcinoma Cells. Oncogene. 1999 Oct 14;18(42):5765-72.

45. Brunetto EL, Teixeira Sda S, Giannocco G, Machado UF, Nunes MT. T3 Rapidly Increases Slc2a4 Gene Expression and Glut4 Trafficking to the Plasma Membrane in Skeletal Muscle of Rat and Improves Glucose Homeostasis. Thyroid. 2012 Jan;22(1):70-9.

46. Kindler S, Wang H, Richter D, Tiedge H. Rna Transport and Local Control of Translation. Annual Review of Cell and Developmental Biology. 2005;21:223-45.

47. Laird-Offringa IA, de Wit CL, Elfferich P, van der Eb AJ. Poly(a) Tail Shortening Is the Translation-Dependent Step in C-Myc Mrna Degradation. Molecular and Cellular Biology. 1990 Dec;10(12):6132-40.

48. Srichai MB. Integrin Structure and Function. In: Zent R, editor. Cell-Extracellular Matrix Interactions in Cancer: Springer Science+Business Media; 2010. p. 19.

49. Moura EG, Moura CC. [Regulation of Thyrotropin Synthesis and Secretion]. Arquivos Brasileiros de Endocrinologia e Metabologia. 2004 Feb;48(1):40-52.

50. Cheng SY, Leonard JL, Davis PJ. Molecular Aspects of Thyroid Hormone Actions. Endocrine Reviews. 2010 Apr;31(2):139-70.

51. Davis PJ, Davis FB, Lin HY, Mousa SA, Zhou M, Luidens MK. Translational Implications of Nongenomic Actions of Thyroid Hormone Initiated at Its Integrin Receptor. American journal of physiology Endocrinology and Metabolism. 2009 Dec;297(6):E1238-46.

52. Ashworth R, Hinkle PM. Thyrotropin-Releasing Hormone-Induced Intracellular Calcium Responses in Individual Rat Lactotrophs and Thyrotrophs. Endocrinology. 1996 Dec;137(12):5205-12.

53. Roussel JP, Grazzini E, Zumbihl R, Rodriguez E, Astier H. Triiodo-L-Thyronine Enhances Trh-Induced Tsh Release from Perifused Rat Pituitaries and Intracellular Ca2+ Levels from Dispersed Pituitary Cells. European Journal of Pharmacology. 1995 Apr 28;289(2):205-15.

54. Rodriguez-Ortiz ME, Canalejo A, Herencia C, Martinez-Moreno JM, Peralta-Ramirez A, Perez-Martinez P, et al. Magnesium Modulates Parathyroid Hormone Secretion and Upregulates Parathyroid Receptor Expression at Moderately Low Calcium Concentration. 
Nephrology, dialysis, transplantation : official publication of the European Dialysis and Transplant Association - European Renal Association. 2014 Feb;29(2):282-9.

55. Kasahara K, Tasaka K, Masumoto N, Nishizaki T, Mizuki J, Tahara M, et al. High Magnesium Concentration Inhibits Ligand-Stimulated Calcium Influx and Hormone Secretion in Rat Pituitary Lactotropes with Involvement of Intracellular Free Magnesium. Biochemical and Biophysical Research Communications. 1993 Nov 30;197(1):92-9.

56. Hong BZ, Kang HS, So JN, Kim HN, Park SA, Kim SJ, et al. Vascular Endothelial Growth Factor Increases the Intracellular Magnesium. Biochemical and Biophysical Research Communications. 2006 Aug 25;347(2):496-501. 